\title{
INFORMAÇÃO, EDUCAÇÃO E COMUNICAÇÃO EM SAÚDE: ANÁLISE DAS CONCEPÇÕES DOS COORDENADORES DAS CAMPANHAS DE VOZ NO DISTRITO FEDERAL
}

\author{
Analysis the conceptions of information, education and health \\ communications among the coordinators of voice campaigns in the \\ Federal District of Brazil
}

Rodrigo Dornelas ${ }^{(1)}$, Maria Fatima de Sousa ${ }^{(2)}$, Ana Valéria Machado Mendonça( ${ }^{(3)}$

\begin{abstract}
RESUMO
Objetivo: compreender e discutir as concepções de informação, educação e comunicação (IEC) em saúde dos fonoaudiólogos que coordenaram e desenvolveram as campanhas de voz em Brasília, nos anos 2009 e 2010. Métodos: pesquisa qualitativa que utilizou roteiro de perguntas semiestruturado que tratava da organização da campanha da voz em Brasília. As entrevistas foram realizadas com os organizadores das campanhas da voz nos anos de 2009 e 2010, totalizando 08 entrevistas. O material das entrevistas foram gravados e posteriormente transcritos, para a análise foram seguidas as etapas da Análise de Conteúdo (AC). A discussão foi efetuada com base no referencial teórico de informação, educação e comunicação em saúde. Resultados: a Informação em Saúde é vista como a descrição do funcionamento do aparelho fonador e não como dados disparadores necessários para o planejamento e execução de ações eficazes para a campanha; a Educação em Saúde, como repasse de informações e não como um processo de construção coletivo a respeito dos cuidados necessários para o bem estar vocal; e, a Comunicação em saúde, limita-se aos espaços da mídia e não como estratégia de construção de comunicação partindo do conhecimento da população a respeito do assunto. Conclusão: o conceito IEC está presente na campanha de voz em Brasília, porém a concepção que a cerca por parte dos responsáveis pela execução e elaboração das ações está longe de um consenso. A dificuldade em falar sobre informação, educação e comunicação em saúde reflete na fragilidade teórica em que a campanha de voz está ancorada.
\end{abstract}

DESCRITORES: Sistemas de Informação; Comunicação em Saúde; Educação em Saúde; Promoção da Saúde; Voz

\section{INTRODUÇÃO}

A Fonoaudiologia, ao completar 30 anos de sua regulamentação, tem buscado ampliar suas ações para o campo da Saúde Coletiva, desenvolve

(1) Universidade Federal de Sergipe - UFS, Lagarto, Sergipe, Brasil.

(2) Universidade de Brasília - UnB, Brasília, Distrito Federal, Brasil.

(3) Universidade de Brasília - UnB, Brasília, Distrito Federal, Brasil.

Fonte de auxílio: CAPES

Conflito de interesses: inexistente práticas de saúde voltadas para a população e, enfatiza, sobretudo a prevenção e a promoção, no âmbito da Educação em Saúde.

Entretanto, sabe-se que as ações educativas nem sempre atingem seus objetivos, uma vez que diversos fatores podem potencializar ou até mesmo dificultar o estabelecimento de uma prática educativa que efetivamente contribua para a transformação na saúde das pessoas. Dentre tais fatores, ressalta-se a formação biomédica dos profissionais da área, a baixa inserção nos serviços públicos de saúde, em especial os de atenção primária, e a incipiente construção de 
conhecimentos que relacionem a Fonoaudiologia ao campo da Saúde Coletiva. Assim, percebe-se que as concepções que envolvem as ações educativas dos fonoaudiólogos encontram alicerce neste contexto ainda hegemônico, em que a saúde é entendida enquanto ausência de doença ${ }^{1}$, o que traz implicações importantes nas ações educativas que são desenvolvidas.

Poucos são os estudos delineados de forma a discutir as concepções e as práticas de informação, educação e comunicação em saúde, adotadas em campanhas promovidas pela Fonoaudiologia, e é nessa perspectiva que este artigo busca iniciar uma reflexão acerca das práticas desenvolvidas nas Campanhas de Voz, que atualmente são consideradas como uma das principais ações promovidas pela área, mobilizando grande parte da comunidade fonoaudiológica. Portanto, tem-se como objetivo deste artigo compreender e discutir as concepções de informação, educação e comunicação em saúde dos fonoaudiólogos que coordenaram e desenvolveram as campanhas de voz em Brasília, nos anos 2009 e 2010.

\section{Cenário: as campanhas de voz}

As campanhas da voz promovidas por fonoaudiólogos tem suas ações organizadas por atores regionais, em diversas localidades. A Sociedade Brasileira de Fonoaudiologia (SBFa) estimula a participação dos profissionais premiando a melhor campanha de voz do ano subsequente, escolhida no congresso anual de Fonoaudiologia. Apesar da SBFa não estabelecer a estratégia a ser utilizada nas campanhas, faz-se necessário, para que a campanha concorra ao prêmio de reconhecimento pelas ações executadas, seguir alguns critérios, como utilizar o tema estabelecido desde 2005, 'Seja Amigo de sua voz", e, em todo o material impresso e digital conter o símbolo da campanha ao lado da logomarca da SBFa (http://www.sbfa.org.br/campanhadavoz/index.htm).

No Distrito Federal, o objetivo da Campanha de voz é "conclamar a população para ações de educação em saúde voltadas para a conscientização vocal e suas implicações na comunicação, na saúde, cultura e em sua qualidade de vida"2. Assim, os responsáveis pela campanha discutem sobre as ações a serem executadas e a estrutura necessária para realizá-las.

Deste modo, a atuação do responsável pela campanha direciona o percurso orientado pelos requisitos propostos pela $\mathrm{SBFa}$ e à demanda regional na qual as ações serão inseridas, tendo autonomia necessária para articular e desenvolver um trabalho contextualizado.
Porém a autonomia exige uma formação relacionada aos principais conceitos trabalhados em uma campanha de saúde. As propostas devem ser relacionadas ao objetivo geral da campanha, o conhecimento prévio da realidade em que ocorrerão tais ações e, principalmente, deve haver uma consonância entre todos os responsáveis para que o foco da campanha não se modifique no decorrer de sua trajetória. Campanhas podem ser instrumentos capazes de gerar modificações no estado de saúde de uma população ${ }^{3}$. Para tanto, as campanhas deveriam fazer parte de atividades programáticas que seguissem um planejamento estratégico, com resultados de curto, médio e longo prazos esperados, produzindo mudanças no cenário da saúde pública ${ }^{3}$.

\section{IEC: Instrumento potencializador nas ações em Saúde}

Há aproximadamente 15 anos, em setembro de 1996, ocorria a 10 ${ }^{\text {a }}$ Conferência Nacional de Saúde. As conquistas do SUS ainda não tinham alcançado os níveis desejados de participação dos diversos segmentos da sociedade. Dessa maneira, os delegados presentes discutiram a proposta que contemplasse ações de informação, educação e comunicação (IEC), voltadas para as exigências inerentes a um novo paradigma na atenção à saúde ${ }^{4}$. Nascia nesse momento, por parte do poder público a reflexão sobre a importância de ações relacionadas a informação, educação e comunicação em saúde 5 .

A informação, no contexto da saúde, tem estado associada à organização de sistemas de dados com o objetivo de apoiar a tomada de decisões, para intervenção em uma dada realidade. Assim, o papel da informação em saúde tem sido entendido como subsídio a essa intervenção e, no entanto, contribui para o entendimento de que a realidade de saúde que traduz, deve influenciar decisões e modificar percepções. A Informação é definida como o conhecimento acrescido de significado e a sua relevância está no tratamento recebido tanto nos seus componentes como em seus conceitos principais 6 .

A Informação em saúde denota premissa essencial na organização de uma campanha ou atividade que tem como fim disparar as questões acerca dos cuidados que se deva ter com a saúde ${ }^{7}$, pois o acesso à informação amplia a capacidade de instrumentalização dos sujeitos nos processos decisórios, ou seja, na construção da Qualidade da Comunicação e Informação em Saúde . $^{2}$

Educação, está na reflexão da realidade do educando, retomando esta realidade posteriormente e assim, com subsídios para transformá-la. 
A educação deve contribuir para formar cidadãos e despertar responsabilidades, devendo ser entendida não apenas como um meio de adquirir conhecimentos, mas também de transformar a realidade do sujeito que é educado ${ }^{9}$.

Desta forma, ressalta-se que o processo de Educação em Saúde, para que seja transformador, deverá partir de uma escuta apurada e assim, realizar a intervenção. Além do escutar ser uma habilidade fundamental na educação em saúde ${ }^{10}$ entende-se que seu objetivo também é de oportunizar momentos de reflexões e ações capazes de possibilitar às pessoas um aprendizado consciente, sem a intenção de controlar suas vidas ${ }^{11}$.

As relações são efetivadas por meio da comunicação, elemento significativo que nos propicia trocas de saberes, discussões e conhecimento. A comunicação em saúde é fator primordial para o sucesso da Promoção de Saúde, há que se considerar a importância da atitude dos profissionais da saúde como aliados na valorização do senso comum e da experiência empírica das populações, beneficiando se assim a incorporação do saber técnico de utilidade pública sem prejuízo do patrimônio cultural que está na base da identidade e da saúde desses grupos ${ }^{12}$.

Considerando tais pressupostos, as políticas de IEC adotadas pelo Ministério da Saúde postulam que ações de informação, educação e comunicação em saúde, quando dissociadas, levam a caminhos turvos, mesmo considerando esses campos distintos de ação em saúde ${ }^{4}$. Porém, é na junção desses campos que o trabalho com a população se concretiza no acesso à informação, numa estratégia de comunicação, visando à educação, num horizonte de exercício da cidadania, contribuindo para a busca da qualidade de vida por meio da participação da população na construção do conhecimento ${ }^{13}$.

\section{MÉTODOS}

A presente pesquisa foi aprovada pelo Comitê de Ética em Pesquisa da instituição de origem sob o número de registro 046/11. Todos os participantes foram informados sobre as condições da pesquisa e assinaram o Termo de Consentimento Livre e Esclarecido. Este estudo atende as disposições da Resolução 196/96 do CNS.

Esse estudo delineou-se como uma pesquisa com abordagem qualitativa. Foi realizado em Brasília, Distrito Federal, com os coordenadores das campanhas de voz nos anos de 2009 e 2010.

Para que pudesse contemplar os objetivos deste estudo, os sujeitos participantes foram escolhidos intencionalmente de acordo com seu papel na campanha de voz, ou seja, os sujeitos responsáveis pela organização e execução das ações das campanhas realizadas no Distrito Federal, tendo participado da definição das informações trabaIhadas, dos materiais utilizados e da metodologia das atividades. Dessa forma, 07 sujeitos foram entrevistados, sendo esse o número equivalente ao universo deste estudo.

Todas as participantes deste estudo são mulheres, graduadas em Fonoaudiologia, com idades entre 25 e 53 anos. A Figura 1 apresenta a caracterização da amostra.

A maioria dos sujeitos está há menos de cinco anos atuando na campanha de voz, sendo esse tempo coincidente, em geral, com um terço do tempo que se graduaram. Todas possuem título de especialista, sendo 04 delas na área de voz.

Para manter o sigilo da pesquisa, os sujeitos participantes foram identificados como S1, S2, S3, S4, S5, S6 e S7. A identificação ocorreu de maneira aleatória, por sorteio e, independe dos dados apresentados na Figura 1, identificados como A,B,C,D,E,F e G.

\begin{tabular}{|c|c|c|c|}
\hline Sujeitos & $\begin{array}{c}\text { Especialização em } \\
\text { voz }\end{array}$ & $\begin{array}{c}\text { Tempo de formação } \\
\text { em Fonoaudiologia } \\
\text { (em anos) }\end{array}$ & $\begin{array}{c}\text { Tempo de atuação na } \\
\text { campanha de voz } \\
\text { (em anos) }\end{array}$ \\
\hline A & Sim & 30 & 10 \\
\hline B & Não & 14 & 4 \\
\hline C & Não & 2 & 4 \\
\hline D & Sim & 22 & 5 \\
\hline E & Sim & 29 & 11 \\
\hline F & Não & 16 & 4 \\
\hline G & Sim & 10 & 4 \\
\hline
\end{tabular}

Figura 1 - Caracterização da amostra de participantes 
As entrevistas foram agendadas previamente por contato telefônico e realizadas individualmente. Depois de lido e assinado o Termo de Consentimento Livre e Esclarecido deu-se início ao procedimento de coleta de dados.

Utilizou-se um roteiro de perguntas semiestruturado que tratava da organização da Campanha de voz. As entrevistas foram gravadas, identificadas com as iniciais das entrevistadas e, transcritas.

Para a análise dos dados, foram seguidas as etapas da Análise de Conteúdo: organização dos dados, codificação, categorização, inferências e interpretação. A categorização inicial correspondeu aos itens previamente estabelecidos no roteiro de entrevista. Dentre as comunicações, os materiais textuais escritos são os mais tradicionais na análise de conteúdo, podendo ser manipulados pelo pesquisador na busca por respostas às questões de pesquisa, nesta direção, outros estudos apontam que a análise de conteúdo é um dos procedimentos clássicos para analisar o material textual ${ }^{14}$. As transcrições das entrevistas foram organizadas em temas seguindo o roteiro semiestruturado aplicado, em seguida categorizadas por assuntos de acordo com a frequência em que surgiam no decorrer das transcrições.

As categorias foram nomeadas após a organização sistêmica do conteúdo transcrito em temas relacionados com o objetivo da pesquisa, à saber: objetivo da campanha da voz; informação em saúde; educação em saúde e comunicação em saúde. Para cada tema identificou-se categorias que delineavam os diferentes pontos de vista sobre determinado tema. Os trechos apresentados são recortes das entrevistas que possibilitaram a identificação em suas respectivas categorias, transcritos de maneira literal à fala do entrevistado.

A discussão dos dados foi efetuada com base no referencial teórico recente de informação, educação e comunicação em saúde ${ }^{5}$.

\section{RESULTADOS}

De modo a esclarecer os objetivos da Campanha de Voz na perspectiva dos sujeitos participantes, tomou-se a seguinte questão como tema disparador: "Para você, qual é o objetivo da campanha de voz?", em que foi possível perceber 3 categorias distintas de respostas apresentadas abaixo. Ressalta-se que o objetivo colocado pela SBFa é promover ações que visem a conscientização da sociedade quanto à importância da saúde vocal em suas diferentes dimensões ${ }^{15}$.

\section{Divulgar a Fonoaudiologia}

Esta categoria evidencia a força do corporativismo de classe profissional nas ações organizadas em prol da saúde da população.

S1 "[...] Divulgar a Fonoaudiologia."

S4 "[...] Orientar a população da... existência da Fonoaudiologia."

S5 "[...] divulgar a importância do fonoaudiólogo e da atuação do fonoaudiólogo nessa área." voz."

S6 "[...] porque o fonoaudiólogo trabalha com

\section{Conscientizar o sujeito}

S2 "[...] pessoas não tem essa consciência

S3 "[...] é conscientizar as pessoas quanto a saúde da voz e quanto aos cuidados que se pode ter pra não prejudicar a voz

\section{Funcionamento normal e alterações fisiológicas da voz}

Informação em Saúde

Esta categoria denota como, na opinião dos organizadores, a informação em saúde é tratada na campanha da voz de Brasília. A informação em saúde é vista como repasse de informação sob a perspectiva de que aquele que ouve não possui nenhum conhecimento prévio de voz ou dos objetivos elencados para a Campanha.

\section{Informação em saúde como sinônimo de} orientação

S3 "[...] orientar a população com relação aos cuidados que ela tem que ter pra ter uma saúde adequada."

S4 e S6 "[...] dizer o que é o trato vocal, etc... e os problemas que pode acometer."

S8 "[...] mostrar o que é a voz em si e quais os problemas que pode afetar."

S1 "[... o funcionamento das pregas vocais, toda a fisiologia."

S5 "[...] como acontece e como né, o que prejudica."

S6 "[...] quais são os prejuízos, quais são os hábitos prejudiciais a nossa voz, a nossa saúde vocal, quais medidas devem ser tomadas, e os profissionais da voz."

S2 "[...] aos cuidados que você deve ter com relação a sua voz, principalmente em relação aos hábitos né (pausa) de... tabagismo, o álcool."

\section{Mecanismos utilizados para que a informação alcance o público alvo}

Aqui, pode-se notar que a informação em saúde é percebida como veículo, ou seja, são as possibilidades de fazer com que um determinado conteúdo alcance os indivíduos. 
S1 "[...] na panfletagem que os alunos de arte, eles iam ate as pessoas, entregavam o panfleto e explicavam do que se tratava."

S3 "[...] próprias palestras de informação."

S7 "[...] workshops."

S8 "[...] Palestras."

S4 "[...] principalmente da mídia, através de entrevista em rádio."

S5 "[...] entrevista em TV."

\section{Educação em Saúde}

Em relação à Educação em Saúde, utilizou-se da seguinte questão: "O que você entende por Educação em Saúde", sendo observadas 03 categorias de respostas que serão apresentadas abaixo.

\section{Orientar a população}

Foi possível perceber que educar, na perspectiva dos entrevistados, está diretamente ligado à ideia de dar orientações acerca dos melhores comportamentos que devem ser adotados pela população para que esta tenha saúde, o que pode ser observado nos fragmentos abaixo:

S5 "[...] pra mim é quando você orienta as pessoas e as pessoas aprendem por causa da sua orientação."

S8 "[...], ela não ta muito voltada pra população, eu não educo uma população, eu acho que eu oriento uma população."

S7 "[...] orientar sobre os cuidados com a saúde de maneira geral."

\section{Informar}

Observa-se que há também uma tendência dos entrevistados em considerar informação e educação enquanto sinônimos, de acordo com os fragmentos apresentados abaixo:

S2 "[...] informar as pessoas acerca das promoções e prevenção em saúde." "[...] As informações foram passadas."

S1 "[...] e com essa educação carregar esse conhecimento pro seu dia a dia e passar a partir de então porque receberam a informação e cuidar melhor da sua voz."

S4 "[...] então assim né informa e a pessoa passa a ter hábitos diferentes, então ela se educou, ela se apropriou de um conhecimento, então ela realmente foi educada pra cuidar da voz."

S6 "[...] É a mesma coisa q informação em saúde, é informar a população sobre os males da saúde."

S7 "[...] - É uma informação muito importante sobre rouquidão não é normal."

S4 "[... mesmas informações que foram passadas no sentido de informação na saúde."
S6 "[...] é você trazer pro, no caso o nosso foco foi a população né, então assim é você trazer pra população os conceitos que eles necessitam saber dentro da área da saúde."

S6 "[...] a população é leiga em relação a isso, você vai compartilhar um pouquinho nesse aspecto."

Os aspectos colocados no que se refere à Educação em Saúde ainda sugerem uma modificação de postura por parte do responsável pela execução das ações na campanha.

S3 "[...] não ficar aquela coisa só o profissional da saúde tem o conhecimento."

S5 "[...] alguma tarefa mais prática no sentido de tentar verificar hábitos, pra tentar a partir daí modificar algum hábito."

\section{Comunicação em Saúde}

Por fim, em relação à Comunicação em Saúde pôde-se observar 2 categorias de respostas, à seguir.

\section{Comunicação Unidirecional}

A categoria traz à tona, dentre outras questões, o papel do profissional de saúde, neste caso, o responsável por executar as ações da campanha de voz. Nesta categoria a hierarquia entre o leigo, que busca informações sobre voz e o detentor da informação, responsável por executar as ações da campanha de voz é observada na maioria das colocações. O reflexo da hierarquia está no modo como é colocada a mensagem aos participantes da campanha. Há uma visível preocupação no que refere ao trajeto necessário para que a mensagem chegue aos participantes, porém a construção da mensagem e sua contextualização não é colocada pelos responsáveis, como pode ser observado nos fragmentos de fala dos entrevistados abaixo:

S1 e S4 "[...] Uso da mídia pra divulgar as ações da campanha

S2 "[...] comunicação acho que se dá de uma forma em geral, em termos de panfletagem, de audiovisual, de cartazes, banners."

S3 "[...] dentro de um próprio hospital você disponibilizar algum material que seja escrito."

S7 "[...] de você estar passando essa informação pra todas as pessoas, pra população de uma forma em geral." "[...] seja a forma deu falar a informação em saúde."

S5 "[...] Dado o seu conhecimento é você estar disponibilizando a ter, um contato, a ter um interlocutor junto com aquela outra pessoa, então mesmo q seja através da mídia, de panfletos, mas de você estabelecer alguma comunicação." 
S6 "[...] comunicar às pessoas e fazer com que as pessoas compreendam a necessidade e os cuidados que deve ter com a voz."

\section{Comunicação Bidirecional}

Esta categoria demonstra a possibilidade da construção do processo de comunicação na campanha de voz de forma contextualizada.

S6 "[...] eu acho que a comunicação talvez seja a parte que você, que é uma parte desse processo, $[. .$.$] tem que ter a ideia do trocar ideia é a hora que$ você ouve o paciente, você fala para ele, não pode tomar leite, ele vira pra você e fala assim: mas eu só tenho leite na minha casa e eu tenho uma vaca e só posso comer isso. Tomar leite e comer queijo e ai eu falo assim, puxa vida, então eu vou tentar ver o que pode fazer pra poder minimizar a situação e chegar onde a gente precisa, uma troca, é muito mais próximo."

\section{DISCUSSÃO}

Se o objetivo colocado pelos organizadores da Campanha de voz é divulgar a Fonoaudiologia, qual a eficácia em relação aos cuidados com a voz para a população que procura tal espaço para saber sobre voz? O questionamento pode ser enfatizado ao acrescentar que a Fonoaudiologia não é a única ciência responsável pelos cuidados com a voz, há pesquisas que relatam experiências positivas de equipes interdisciplinares com voz ${ }^{16}$. Mesmo a campanha de voz sendo idealizada por uma entidade de classe, é necessário esclarecer à população sobre todas as possibilidades relacionadas à voz, tema da campanha. Ter como objetivo divulgar a Fonoaudiologia interfere no processo de construção das ações e distorce os propósitos de uma campanha educativa em saúde. Se o objetivo é divulgar uma profissão, a problematização a respeito da voz e seus cuidados se torna coadjuvante, assim as ações terão como foco uma disputa por território profissional, a divulgação das ações que somente a Fonoaudiologia pode realizar para a população no que se refere à voz, excluindo a importante atuação de outros profissionais. A visão estreita, enfocando a atuação profissional em detrimento ao cuidado com a voz que se propõe a realizar em uma campanha, modifica as ações, as convicções, os objetivos e os resultados esperados.

Conscientizar a população, uma das categorias que surgiram relacionadas ao objetivo da campanha de voz é uma prática recorrente quando se refere a intervenções em massa. O termo "conscientizar" aparece em diversos espaços, como prioridade no trabalho junto à população, e serve também como sinônimo de modificação de comportamento e/ou atitudes negativas e, estando presente no objetivo proposto pela SBFa para a campanha de $\mathrm{voz}^{15}$.

A discussão do diálogo libertador ${ }^{17}$ coloca a constante capacidade de transformação, na qual o homem assume um papel de sujeito com atitude crítica, reflexiva e histórica e o conhecimento e entendido como inacabado, progressivo e contínuo. Nesse sentido, a realidade é entendida como um processo modificável a partir da conscientização como teoria e prática da libertação ${ }^{17}$. Na teoria da conscientização, é destacado que por meio da crença em si mesmo o homem é capaz de dominar os instrumentos de ação à sua disposição, ou seja, que por meio de suas práticas o homem torna-se um importante agente transformador do seu universo social, cultural e histórica. A partir dessas colocações fica evidente a relevância da conscientização para o ser humano, frente às suas possibilidades de intervenções na resolução de problemas que tendem a afetar a sociedade, tal como, a conscientização para a prática dos cuidados com a voz. Porém a conscientização não deve ser vista apenas como responsabilidade da população, mas o processo de conscientização deve ser construído e elaborado coletivamente, e deve ir além da simples instrumentalização sem questionar os determinantes envolvidos nos processos saúde-doença-cuidado.

Em relação à Informação em Saúde, questionou-se aos sujeitos como estes a entendem, e pôde-se observar a dificuldade dos mesmos em sintetizarem seus pensamentos acerca da Informação em Saúde.

A dificuldade em discutir informação em saúde demonstrada por parte dos entrevistados revela a fragilidade quando o tema é elucidado no contexto das campanhas em saúde. A informação em saúde é algo necessário para que a campanha tenha subsídios teóricos no embasamento da construção de materiais educativos e de comunicação.

Atualmente, nas discussões sobre informação em saúde, a preocupação que vem à tona é a integração dos sistemas de informação para que se possa mapear, esclarecer e propor estratégias específicas para determinado local/população. Desta maneira, a integração dos sistemas beneficia a população com estratégias relacionadas, contextualizadas de acordo com a realidade local, subsidiando a atuação do profissional da saúde.

Anterior a essa discussão de integração dos sistemas, a campanha da voz promovida por fonoaudiólogos, para que possa contemplar os objetivos propostos é necessário se beneficiar da informação em saúde. Utilizar os dados disponíveis pelos órgãos competentes faz com que a campanha alcance um maior número de indivíduos interessados pelo tema e com necessidades de 
compartilhar conhecimentos sobre o assunto. Deste modo o poder público é colocado como parceiro na disponibilização de tais dados e, caso não haja dados populacionais que abranjam a campanha da voz, faz-se necessário estudos para elaboração de banco de dados que priorizem os aspectos vocais da população.

O percurso midiático da informação é o principal aspecto elencado nesta categoria. O papel da informação em saúde, de subsidiar a construção de um conhecimento ou até mesmo de sustentar a identificação da população alvo da campanha com os aspectos elencados como prioridade pelos responsáveis, se modifica quando a prioridade é o percurso traçado para a informação $0^{18}$ e não a informação em saúde como objetivo de potencialização da comunicação e educação em saúde.

Metodologicamente, Educação em Saúde enfoca a relação entre usuário/paciente/cliente e profissional da saúde. Tal relação remete diretamente ao processo de educação. O lugar que os atores ocupam é tema de diversos artigos, pois o "segredo" da eficácia dessa relação está no posicionamento que o profissional de saúde toma para $\mathrm{si}^{10}$. Neste sentido, considera-se como um dos mais importantes fatores que influenciam neste processo de construção do aprendizado.

O responsável pela campanha de voz é visto como orientador. Aquele que orienta a população para o caminho correto. O termo orientar remete a guiar, encaminhar, dirigir o que difere de Educação em Saúde que preza por uma escuta antecedida à qualquer ação pretendida. O idealizador das campanhas deve desenvolver habilidades para ouvir as narrativas da população, com uma escuta qualificada, o que implica em lembrar que a narração de um fato pode modificar a maneira de encará-lo e agir sobre determinada situação ${ }^{19}$. Assim, o processo de Educação em Saúde, para que seja transformador, deverá partir de uma escuta apurada e à partir daí deve-se intervir ${ }^{10}$.

A Educação em saúde é colocada como informar a população sobre determinada situação. Neste contexto compreende-se educação em saúde como um encontro entre dois lados (profissional de saúde e usuário) sustentado pelo diálogo e a troca de experiências, envolvendo intencionalidades educativas, não se restringindo às informações, orientações e ações com ênfase somente na técnica ${ }^{20}$.

Um dos maiores desafios de quem trabalha com educação em saúde é superar os limites da comunicação meramente informativa. Educar é mais do que transmitir uma informação. É formar: transmitir ideias, visões de mundo, valores, atitudes. Isso pressupõe o aprendizado como uma via de mão dupla, numa concepção crítica e problematizadora, em que ambos, professor e aluno são aprendizes, num processo de trocas permanentes e transformação política da realidade ${ }^{21}$.

Há possibilidades de atividades práticas direcionadas ao cotidiano e deslocamento da relação de hierarquia entre o detentor do conhecimento e a população dita "leiga". A Educação em Saúde se faz com trocas, à partir da validação ou reconhecimento do saber que a população tem sobre saúde ou o tema abordado, neste caso sobre voz.

Nota-se que a descrição do entendimento de comunicação em saúde é colocado como um processo de transmissão de mensagem, ou seja, a maneira como se faz a mensagem chegar no interlocutor, neste caso a população.

O processo utilizado remete ao modelo desenvolvimentista de comunicação, introduzido no Brasil por volta de 1950. Este modelo concebe a comunicação como moldagem de atitudes e comportamentos, considera a comunicação como unidirecional e linear, como passagem da mensagem de um pólo para o outro ${ }^{22}$. Tal modelo dificulta a contemplação de aspectos contextuais de acordo com os espaços em que as ações da campanha são realizadas. A problematização de como a mensagem chegará até à população é necessária, porém atentar-se à construção dessa mensagem com a população é essencial. O público atingido pela campanha de voz é plural, há uma heterogeneidade significante que necessita ser assistida pelas ações estabelecidas, desta maneira, a mensagem a ser construída deve ser plural, deve contemplar ao que buscam por informações sobre os cuidados com a voz.

A comunicação em saúde, para ser efetiva, deve ser construída com a população foco. A necessidade de se realizar um discurso que possa ser ouvido, que as pessoas se identifiquem, faz com que a ação proposta seja eficaz. Para isso, se faz necessário, construir coletivamente o conhecimento, deve se atentar ao contexto em que a população esta inserida.

\section{CONCLUSÃO}

As tecnologias de IEC estão presentes na campanha da voz em Brasília, porém a concepção que a cerca por parte dos responsáveis pela execução e elaboração das ações está longe de um consenso, dado observado nas entrevistas. A dificuldade em falar sobre informação, educação e comunicação em saúde reflete na fragilidade teórica em que a campanha de voz está ancorada. A dificuldade conceitual faz reflexo no objetivo que norteia a campanha na concepção dos organizadores. Objetivos distintos para a campanha são apresentados de modo a se perder o foco do objeto 
da campanha, ora apresentado como divulgar uma profissão, ora como demonstrativo da importância da atuação profissional do fonoaudiólogo. Neste caso, o papel das campanhas de saúde como ferramenta instrumentalizadora da população se enfraquece, pois nesta situação as questões relacionadas à voz ficam em segundo plano.

De modo geral, a Informação em Saúde é vista como a descrição do funcionamento do aparelho fonador e não como dados disparadores necessários para o planejamento e execução de ações eficazes para a campanha; a Educação em Saúde é colocada como repasse de informações e não como um processo de construção coletivo do conhecimento a respeito da voz e os cuidados necessários para o bem-estar vocal e, a Comunicação em saúde, limita-se aos espaços da mídia, como exemplo as entrevistas em rádios e TVs e não como uma estratégia de construção de comunicação partindo do conhecimento da população a respeito do assunto, realizando também intervenções em rádios comunitárias e em grupos reconhecidos pela população regionalmente.

A campanha de voz no Distrito Federal apresenta uma das maiores abrangências no Brasil, segundo dados apresentados no $19^{\circ}$ Congresso Brasileiro de Fonoaudiologia. É necessário otimizar a campanha, com objetivo claro e que a população seja a maior beneficiada com as ações propostas. Faz-se necessário que os responsáveis pela campanha se articulem teoricamente para que possam contemplar os objetivos de uma campanha em saúde, ancorados na sistematicidade que a teoria de IEC possibilita.

\begin{abstract}
Purpose: to understand and discuss the conceptions of information, education and health communication (IEC) of speech therapists who coordinated and developed the Voice Campaigns in Brasilia, in the years 2009 and 2010. Methods: the study used a qualitative approach in which semi-structured questions were used to collect data. The data analysis followed the steps of content analysis and the discussion was based on information, education and health communication theories. Results: it showed that, health Information is seen only as the description of the vocal tract functions and not as data that could be helpful triggers for planning and implementing effective action for the Voice campaigns; the Health Education is seen as a transference of information and not as a collective process with regards to the needed care for vocal and well-being; and Communication in health is limited to press media and not as a strategy for construction of a communication based on the population's knowledge about the subject. Conclusion: the IEC technologies can be observed in the campaigns studied, but the conceptions involved in this process are still far from a consensus. The difficulty in talking about information, education and health communication reflects a fragility of the voice campaigns.
\end{abstract}

KEYWORDS: Information Systems; Health Communication; Health Education; Health Promotion; Voice

\section{REFERÊNCIAS}

1. Cardoso AS, Nascimento MC. Comunicação no Programa Saúde da Família: o agente de saúde como elo integrador entre a equipe e a comunidade. Ciênc. Saúde coletiva. 2012;15(1):1509-20.

2. Associação Profissional dos Fonoaudiólogos do Distrito Federal: Diretrizes da Campanha de voz no Distrito Federal, 2009. Disponível em: http://vozdebrasilia.blogspot.com. Acesso em: 14 mar.2009.

3. Saraceni V, Leal MC, Hartz ZMA. Avaliação de campanhas de saúde com ênfase na sífilis
Congênita: uma revisão sistemática. Rev. Bras. Saúde Matern. Infant. 2005;5(3):263-73.

4. Brasil. Ministério da Saúde. Conselho Nacional de Saúde: Anais da X Conferência Nacional de Saúde, 1996. Disponível em: http://conselho.saude. gov.br/biblioteca/Relatorios/relatorio_10.pdf Acesso em 10 nov. 2011.

5. Brasil. Ministério da Saúde: Informação, Educação e Comunicação para o SUS, 1996. Disponível em: http://bvsms.saude.gov.br/bvs/ publicacoes/cd08_11.pdf Acesso em 05 ago.2010.

6. Nascimento EMR, Costa MC, Mota ELA, Paim JS. Estudo de fatores de risco para óbitos de menores de 
um ano mediante compartilhamento de bancos de dados. Cad. Saúde Pública. 2008;24(11):2593-602.

7. Viacava F, Dachs N, Travassos C. Os inquéritos domiciliares e o Sistema Nacional de Informações em Saúde. Ciência \& Saúde Coletiva. 2006;11(4):863-9.

8. Silva AX, Cruz EA, Melo V. A importância estratégica da informação em saúde para 0 exercício do controle social. Ciência \& Saúde Coletiva. 2007;12(3):683-8.

9. Pinafo E, Nunes EFPA, Gonzalez AD, Garanhani ML. Relações entre concepções e práticas de educação em saúde na visão de uma equipe de saúde da família. Trab. educ. saúde. 2011;9(2):201-21 .

10. Boehs AE, Monticelli M, Wosny AM, Heidemann IBS, Grisotti M. A interface necessária entre enfermagem, educação em saúde e o conceito de cultura. Texto Contexto Enferm. 2007;16(2):307-14. 11. Pereira AV, Vieira ALS, Filho AA. Grupos de educação em saúde: aprendizagem permanente com pessoas soropositivas para o HIV. Trab. Educ. Saúde. 2011;9(1).

12. Rozemberg B. O saber local e os dilemas relacionados à validação e aplicabilidade do conhecimento científico em áreas rurais. Cad. Saúde Pública. 2007;23(1):97-S05.

13. São Paulo. Prefeitura de Osasco. Secretaria de políticas públicas: Ações e estratégias de IEC implantadas no município de Osasco, 2010.

14. Mozzato AR, Grzybovski D. Análise de conteúdo como técnica de análise de dados qualitativos no campo da administração: potencial e desafios. RAC. 2011;15(4):731-47.

15. Sociedade Brasileira de Fonoaudiologia: Manual de sugestões e condutas para realização da Campanha da Voz, 2011. Disponível em: http:// www.sbfa.org.br/campanhadavoz/index.htm. Acesso em 10 jan.2012.

16. Nemr K, Amar A, Abrahão M, Leite GCA, Köhle $J$, Santos $A O$ et al. Análise comparativa entre avaliação fonoaudiológica perceptivo-auditiva, análise acústica e laringoscopias indiretas para avaliação vocal em população com queixa vocal. Revista Bras Otorrinolaringol. 2005;71(1):13-7.

17. Freire P. Conscientização: teoria e prática da libertação - uma introdução ao pensamento de Paulo Freire. $3^{\underline{a}}$ ed. São Paulo. Editora Moraes; 1980.

18. Oliveira MS, Paiva LHC, Costa JV, Neto AMP. Adaptação para o português de questionário de avaliação da informação científica em saúde na imprensa (Index of Scientific Quality). Rev. Bras. Ginecol. Obstet. 2009;31(12):592-7.

19. Teixeira MLO, Ferreira MA. Cuidado compartilhado: uma perspectiva de cuidar do idoso fundamentada na educação em saúde. Texto contexto - enferm. 2009;18(4):750-8.

20. Renovato RD, Bagnato MHS. Práticas educativas em saúde e a constituição de sujeitos ativos. Texto contexto - enferm. Florianópolis. 2010;19(3):554-62.

21. Rangel-S ML. Dengue: educação, comunicação e mobilização na perspectiva do controle-propostas inovadoras. Interface. 2008;12(25):433-41.

22. Oliveira AMF. Informação, educação e comunicação em saúde: é suficiente atuar em rede? Os desafios éticos da formação do profissional de saúde na pós - modernidade. Actas de Saúde Coletiva. 2009:3(1):14-27. http://dx.doi.org/10.1590/1982-0216201412212

Recebido em: 22/05/2012

Aceito em: 24/11/2012

Endereço para correspondência:

Rodrigo Dornelas

SQN 314 Bloco B Ap 107 Asa Norte

Brasília - DF

CEP: 70767-020

E-mail: rodrigodornela@uol.com.br 